\title{
Intraosseous Vascular Access for Alert Patients
}

Stacie Hunsaker

Brigham Young University - Provo, stacie-hunsaker@byu.edu

Follow this and additional works at: https://scholarsarchive.byu.edu/facpub

Part of the Nursing Commons

\section{Original Publication Citation}

AJN, American Journal of Nursing: November 2013 - Volume 113 - Issue 11 - p 34-39 doi:

10.1097/01.NAJ.0000437110.65929.70

\section{BYU ScholarsArchive Citation}

Hunsaker, Stacie, "Intraosseous Vascular Access for Alert Patients" (2013). Faculty Publications. 1550.

https://scholarsarchive.byu.edu/facpub/1550

This Peer-Reviewed Article is brought to you for free and open access by BYU ScholarsArchive. It has been accepted for inclusion in Faculty Publications by an authorized administrator of BYU ScholarsArchive. For more information, please contact ellen_amatangelo@byu.edu. 
Intraosseous Access for Alert Patients: When is it Appropriate?

April 20, 2012 
Intraosseous Access for Alert Patients: The Hows and Whys

A three-year-old female presented to the emergency department (ED) with her parents after experiencing three days of fever, nausea, vomiting, and diarrhea. She was alert but pale, with a central capillary refill of 3 to 4 seconds. The ED physician assessed the patient and ordered some typical lab tests for an ill child: a CBC, electrolytes, and blood cultures. Due to her obvious dehydration, and intravenous (IV) fluid bolus of $20 \mathrm{cc} / \mathrm{kg}$ of warmed normal saline (NS) was ordered. Clearly, care of this little girl at this point was challenging because of her dehydrated state. Additionally, the ED was flooded with patients, and there was only one physician to evaluate several critically-ill patients. This particular day was the kind every ED nurse dreads—no breaks, no reprieve.

Meanwhile, we had a very sick little girl in our charge. Two attempts at IV access were made by the ED nurse. The patient was chubby and dehydrated, clearly a very bad combination for successful IV access. Due to the department's protocol of a maximum of two IV attempts on a pediatric patient per provider, our team called for a pediatric nurse expert's help, and a team of three pediatric nurses quickly arrived. However, this expert team was also having trouble obtaining IV access. They were convinced there was a vein somewhere that was accessible. Over the course of an hour, the pediatric team was unable to obtain vascular access. Subsequently, the patient was deteriorating. Though the nurses were aiming to give quality patient care, time had gotten away from them and the patient was deteriorating. Her skin was beginning to mottle, hypotension had developed, and her heart rate had increased from 148 beats per minute (bpm) to $170 \mathrm{bpm}$.

Once it was apparent traditional venous access was not going to be established, the primary nurse informed the physician. It was determined that immediate IV fluids were crucial. 
The patient required an immediate fluid bolus to prevent her progression from compensated shock to decompensated shock. However, there was one problem--the patient was awake. Although her level of consciousness (LOC) was decreased, she was still somewhat alert and responsive. Could we attempt intraosseous (IO) access and infuse fluids on a patient who was not unconscious? How would we explain to the parents at the bedside that we were going to drill a hole in their baby's leg to infuse fluid? But the patient was deteriorating quickly; all barriers needed to be moved aside to save her life. The decision was made to immediately place an IO in the proximal tibia using the EZ-IO intraosseous drill. The patient rapidly needed a fluid bolus.

ED nurses are frequently confronted with the difficult situation of dehydrated pediatric patients or patients of any age with veins that are difficult to visualize. IV access can be complex when the patient is in any state of shock. How long is it acceptable to wait for the best IV nurse or technician to attempt IV access? How many IV attempts are reasonable in a very ill patient? Are we afraid to use IO needles for vascular access?

IO use is certainly not new to medicine. Dr. C.K. Drinker first reported IO infusion in 1922 (Drinker, 1922). During World War II, IO access was widely used for rapid resuscitation in hemorrhaging patients. Researchers have discovered that fluids and drugs administered via the IO route reach the central circulation as fast as central venous catheters (Hoskins, Zacharia, Copper, \& Kramer, 2007). The American Heart Association (AHA) renewed interest in IO use with the Pediatric Advanced Life Support (PALS) guidelines in the 1980s. Since that time, the AHA has continued to recommend the use of IO access earlier in the care of critical patients.

The AHA and the American Academy of Pediatrics recognize IO cannulation as a simple and effective mode of establishing vascular access in all patients. IO access can be achieved in 
patients of all ages. It provides a non-collapsible vascular space where fluids and medications may be delivered rapidly, reliably, and safely. PALS recommendations include using IO access as the "initial vascular access in severe shock or vasoconstriction and when peripheral IV access cannot be quickly achieved in a child with compensated or hypotensive shock" (American Heart Association \& American Academy of Pediatrics, 2011, pp. 109-111).

Moreover, the Emergency Nurses Association (ENA) and the American Association of Critical Care Nurses (AACN) have endorsed a position paper authored by the Infusion Nurses Society (INS) which clarifies that "a qualified registered nurse may insert, maintain, and remove IO access devices. Intraosseous access should be considered if IV access cannot be obtained, and substantial concern exists for increased morbidity or even mortality in the patient from not obtaining treatment" (Infusion Nurses Society, 2009, p. 187).

Additionally, the American College of Emergency Physicians (ACEP) is supportive of IO cannulation being performed by non-physician practitioners. "There are situations in the emergency department when standard intravenous access procedures fail or are insufficient to meet the clinical needs of the patient. Alternative access methods must be available under such circumstances and their usage should be a part of the standard emergency medicine practice privileges. These alternate access modalities include, but are not limited to, intraosseous lines, external jugular lines, and illumination device guided placement of peripheral lines"(American College of Emergency Physicians, 2011).

Obviously peripheral IV access can be frustrating and time consuming, especially in hypovolemic, critically-ill patients whose veins are constricted, volume-depleted, or damaged by chemotherapy or vascular disease. IO access has been proven to allow rapid and reliable access to central circulation (Frascone, Jensen, Kaye, \& Salzman, 2007). Studies in adult patients 
support that IO insertion is fast and effective. In particular, it has been shown to be a more rapid method of vascular access in patients in a resuscitative state (Leidel, Kirchhoff, Braunstein, Biberthaler, \& Kanz, 2011). For example, Lamhaut et al., provided research which demonstrated IO access was gained within 40 seconds compared with over one minute of peripheral IV access (2010). Further, pediatric patients usually cause caregivers a higher level of anxiety due to their small size. A study using the EZ-IO vascular device showed that successful insertion and infusion were achieved in 94 percent of the patients. Insertion time was ten seconds or less in 77 percent of the one-attempt successful cases (Horton \& Beamer, 2012; Frascone, Jensen, Kaye, \& Salzman, 2007).

The decision to use IO infusion, however effective, must be coupled with concern for the patient's condition. Can an IO device be tolerated in a patient who is conscious? Can IV fluid boluses be infused without causing pain? During IO placement, stimulation of the nociceptors in the skin and periosteum cause pain (Nutbeam \& Daniels, 2010). Patients liken this pain to a large-bore peripheral IV access (Philbeck, Miller, \& Montez, 2009). Because the intraosseous space contains many nerve fibers which are pressure-sensitive, the infusion of fluids or medications may cause pain in a conscious patient. Fortunately, studies have shown that pain can be controlled with the use of 2 percent preservative-free lidocaine injected slowly through the IO device into the marrow space (Philbeck, et al., 2009; Philbeck, Miller, Montez, \& Puga, 2010).

When the IO was initiated through the skin and bone of this three-year-old patient, there was no withdrawal to pain and a pain scale of 0 was noted on the FLACC scale (Merkel, Shayevitz, Voepel-Lewis, \& Malviya, 1997). Once the initial flush was administered, the patient exhibited a pain score of 6 out of 10 on the FLACC scale. After the first fluid bolus was 
initiated, the patient withdrew her leg to pain and grimaced, but after approximately 5 seconds she appeared to relax. At the time of this little girl's treatment, our institution did not have an existing guideline for the use of lidocaine IO prior to the insertion of fluids in a conscious patient. As a result of the critical state of this patient, there was not time for the staff to investigate the use of appropriate lidocaine dosing prior to IV fluids. It was imperative the fluid boluses be instituted quickly. The patient's most significant pain appeared to be on infusion of the initial IV flush and appeared to decrease during the administration of the first fluid bolus.

Our little patient recovered well. After receiving three $40 \mathrm{ml} / \mathrm{kg}$ of warm saline boluses, her heart rate dropped to $110 \mathrm{bpm}$, her blood pressure returned to a normal range, and her capillary refill decreased to $<2$ seconds. She became more alert and responsive. Consequently, she required a 24-hour hospitalization, but recovered with no long-lasting effects from her dehydration.

As a result of this scenario, nursing staff were troubled after witnessing this patient's discomfort. This event stimulated discussion of developing a guideline for the administration of lidocaine in conscious patients. We used this experience to improve our clinical practice regarding difficult vascular access. IO access is now initiated earlier in the care of critical patients who require IV access. The lidocaine guideline was written to be used for alert patients who need pain control for IO fluid infusions (see Figure 1). A pharmacist and ED nurse educator collaborated and developed an order set that a physician or licensed independent practitioner may sign and complete quickly in the event of the immediate need for IO access on a patient who is awake and perceives pain. Two contraindications exist for the use of lidocaine in pediatric patients. In addition to an obvious allergy, a history of seizures or acute seizures in a patient would prohibit the use of lidocaine in this protocol. Lidocaine has the potential to induce 
seizures in infants, children, and adults. Adults reach a toxic level at concentrations greater than $5 \mathrm{mcg} / \mathrm{ml}$, but pediatric toxicities have been reported at much lower concentrations. Lidocaine lowers the seizure threshold and greatly increases the risk of a seizure in pediatric patients who have a history of seizures or acute seizure activity (Moran, Hossain, \& Insoft, 2004; DeToledo, Minagar, Lowe, \& Merredith, 2002).

In summary, this case demonstrates the value of IO access in all patient types-conscious or unconscious. In order for IO use to be accepted and utilized, it is essential for institutions to develop protocols and guidelines for its use. Despite recommendations from specialty organizations such as AHA, ENA, AACN, INS, and ACEP, the use of IO devices are underutilized (Voigt, Waltzman, \& Lottenberg, 2008). Nurses must be actively engaged in the acceptance and use of IO devices. IO access will be successful when a department has a champion who can support and train staff, persuade physician involvement, and maintain staff competency in the use of the tool.

The EZ-IO intraosseous system has been the IO of choice at our institution for several years. It is simple to use and maintain competency. The system uses a hand-held power drill to drive a hollow drill-tipped needle into the intraosseous space. The needles come in three different sizes for varying tissue depth. Our practitioners feel comfortable and confident with the ease of use of the drill. It is precise and drills well even in strong, dense adult bone (see Figure $1)$.

In addition to the EZ-IO device, different types of IO tools are available. The most common are manually inserted devices that are placed by using a twisting and pressing motion. These are most often used in younger pediatric patients due to their softer bones and ease of placement. Manual devices are difficult, if not impossible, to use on adolescents and adults 
because of their calcified bone matrix (see Figures 2 and 3). Spring-loaded devices are available for all ages and sizes (see Figure 4). These are available in adult and pediatric sizes and work by a powerful spring firing the needle into the medullary space.

The preferred placement site for an IO in pediatric patients is the medial proximal tibia, approximately $2 \mathrm{~cm}$ below the tibial tuberosity. Each different IO device has specific approved sites, and different institutions may have specific protocols for IO sites. Other locations for IO placement include the distal femur, the distal tibia directly above the medial malleolus, the humeral head, and the iliac crest. The practitioner must be aware of the approved manufacturer sites to ensure adequate and appropriate placement. 


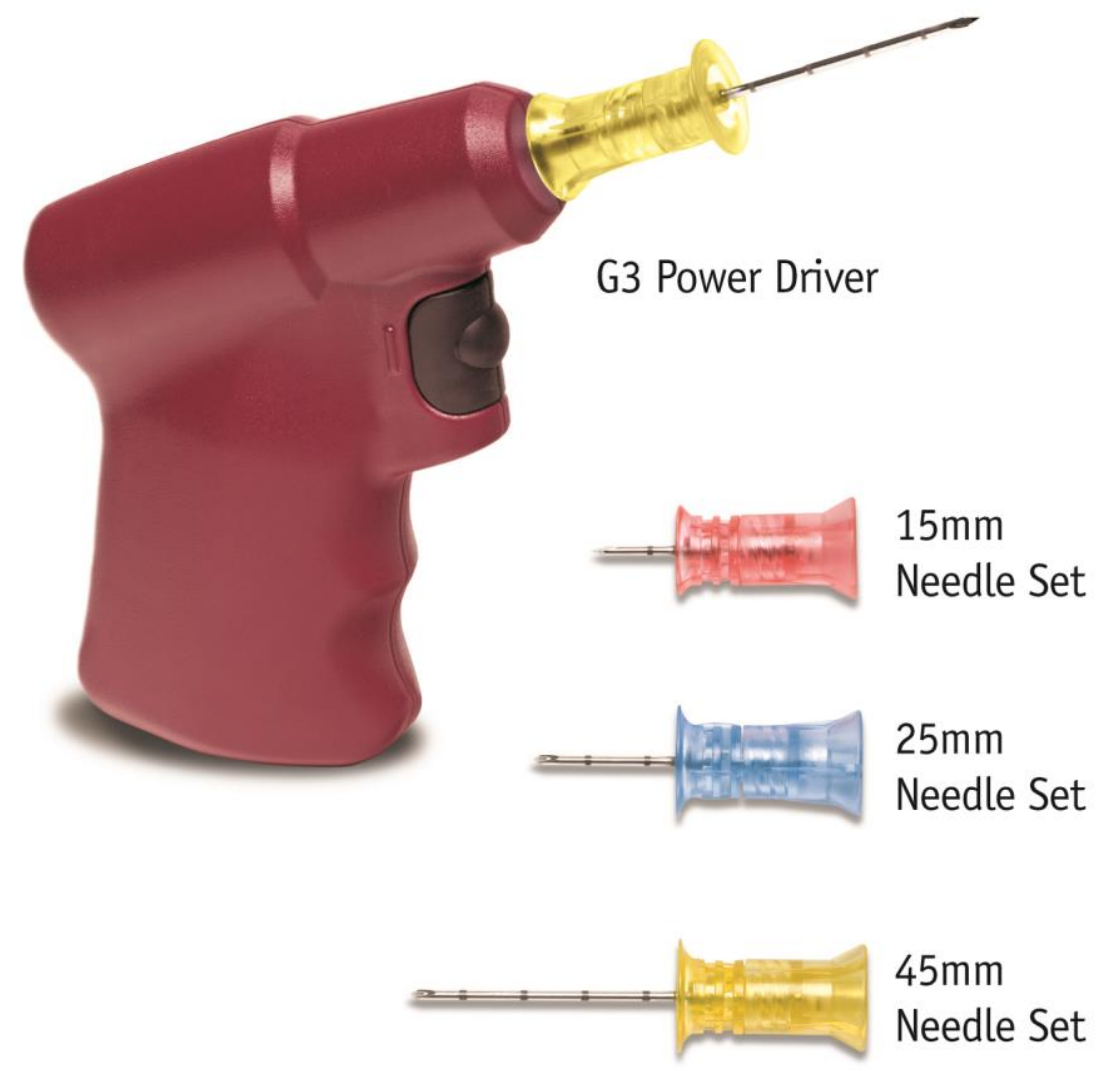

Figure 1. The EZ-IO power driver and needles. 
There are different manual intraosseous needles available for pediatric use. They all have removable trocars to prevent plugging the needle with bone fragments. The handles are made to allow pushing and rotating through the dense periosteum.

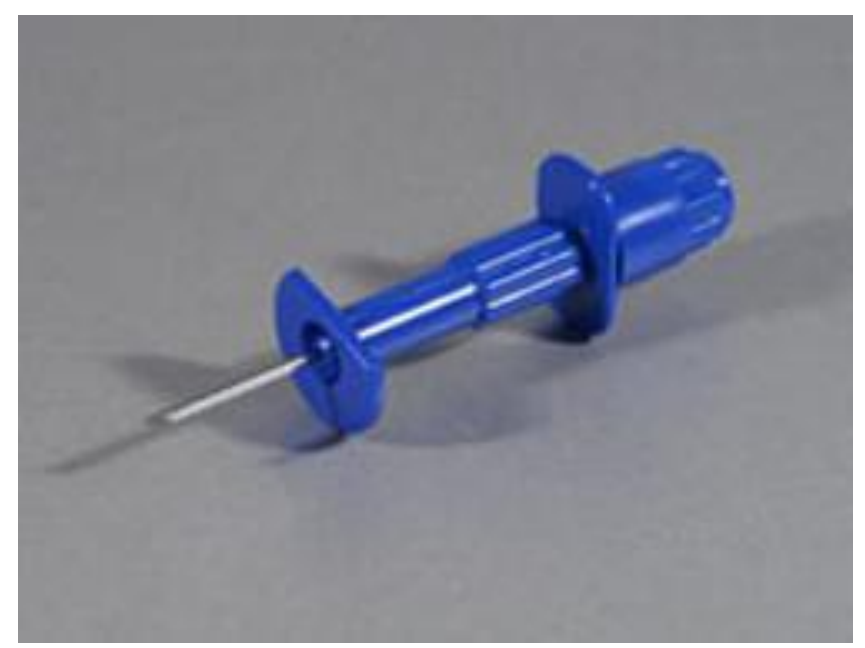

Figure 2. The Jamshidi manual intraosseous device.

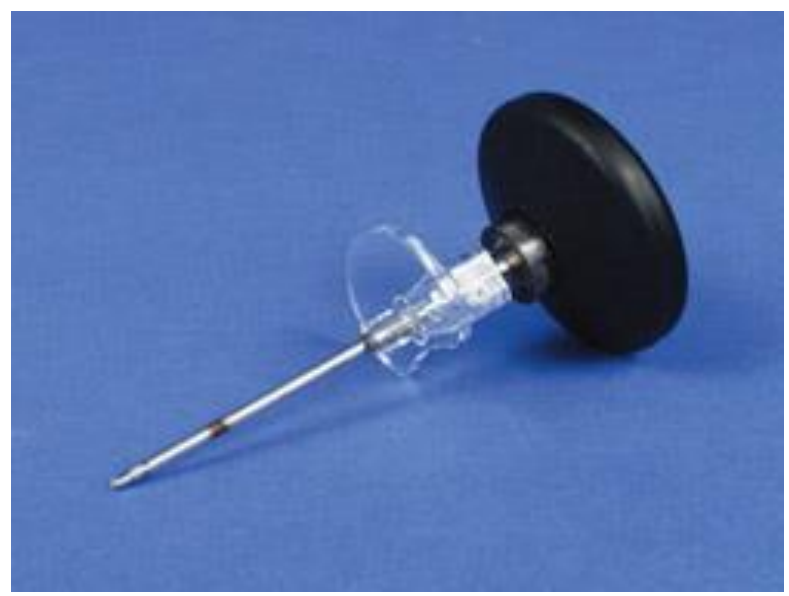

Figure 3. The Cook manual intraosseous device. 
INTRAOSSEOUS ACCESS

11

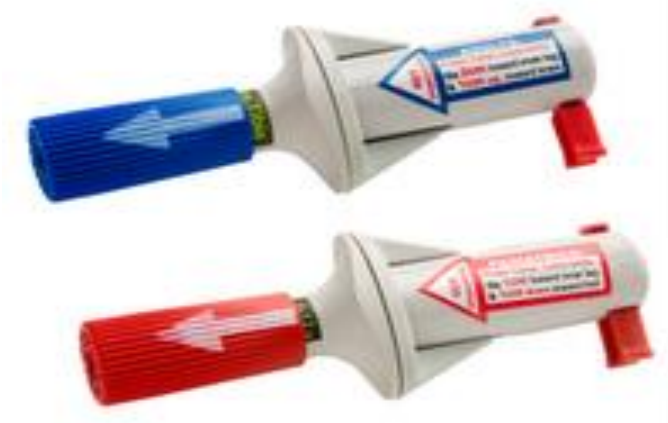

Figure 4. The BIG (bone injection gun.) Adult (blue) and pediatric (red) models. 


\section{PURPOSE}

This procedure describes a process for nursing and/or pharmacy personnel* to administer lidocaine through an intra-osseous (IO) catheter to decrease infusion-related pain in a conscious patient. 10 insertion may cause mild pain in conscious patients, but IO infusions may cause severe discomfort. Lidocaine is meant to be used as an anesthetic and not as analgesia.

For PEDIATRIC patients who may or are able to perceive pain after the IO device is placed and position has been confirmed and secured. CONTRAINDICATED in pediatric patients with acute seizures or history of non-febrile seizures.

1. May give $0.5 \mathrm{mg} / \mathrm{kg}$ (maximum $20 \mathrm{mg}$ ) or $2 \%$ lidocaine (without preservatives or epinephrine) 10 as a slow bolus.

2. Diluted with normal saline to a total volume of $1 \mathrm{ml}$ (see Table 1 below).

3. Wait at least 30 seconds, then flush with $5 \mathrm{~mL}$ of normal saline.

4. If necessary, Step 1 may be repeated as needed to maintain anesthetic effect (do NOT exceed $3 \mathrm{mg} / \mathrm{kg} / 24 \mathrm{hr}$ ).

The volume of lidocaine recommended in pediatric patients is not enough to prime the tubing. A small amount of normal saline is used to ensure the volume is the correct amount to prime the tubing and complete the lidocaine flush. Because of the familiarity and ease-of-use of the Broselow system, we based our lidocaine chart (see Table 1 below) on the weight-based tape recommendations.

ADULT patients: For patients who may or are able to perceive pain after the IO device is placed and position has been confirmed and secure.

1. May give $20-40 \mathrm{mg}(1-2 \mathrm{~mL}$ ) or $2 \%$ lidocaine (without preservatives or epinephrine) $\mathrm{IO}$ as a bolus over 1 minute.

2. Wait at least 30 seconds, then flush with $10 \mathrm{~mL}$ of normal saline.

4. If necessary, Step 1 may be repeated as needed to maintain anesthetic effect (do NOT exceed $3 \mathrm{mg} / \mathrm{kg} / 24 \mathrm{hr}$ ).

* Medication must be ordered by physician or LIP. 


\begin{tabular}{|c|c|c|c|c|}
\hline $\begin{array}{l}\text { Broselow } \\
\text { Color }\end{array}$ & $\begin{array}{l}\text { Weight } \\
\text { (KG) }\end{array}$ & $\begin{array}{c}0.5 \mathrm{mg} / \mathrm{kg} \\
\text { Lidocaine } \\
(\mathrm{mg})\end{array}$ & $\begin{array}{c}20 \mathrm{mg} / \mathrm{ml} \\
\text { Lidocaine } \\
(\mathrm{ml})\end{array}$ & $\begin{array}{c}\text { Normal } \\
\text { Saline } \\
\text { (ml) } \\
\end{array}$ \\
\hline Grey & 3 & 1.5 & 0.08 & 0.92 \\
\hline Grey & 4 & 2 & 0.1 & 0.9 \\
\hline Grey & 5 & 2.5 & 0.13 & 0.87 \\
\hline Pink & $6-7$ & 3.4 & 0.17 & 0.83 \\
\hline Red & $8-9$ & 4.25 & 0.21 & 0.79 \\
\hline Purple & $10-11$ & 5.25 & 0.26 & 0.74 \\
\hline Yellow & $12-14$ & 6.5 & 0.33 & 0.67 \\
\hline White & $15-18$ & 8.25 & 0.41 & 0.59 \\
\hline Blue & $19-22$ & 10.37 & 0.52 & 0.48 \\
\hline Orange & $24-28$ & 13 & 0.65 & 0.35 \\
\hline Green & $30-36$ & 16.5 & 0.83 & 0.17 \\
\hline
\end{tabular}

This table represents approximate dosing based on Broselow's weight breakpoints.

Table 1. IO Lidocaine dosing chart. 


\section{References}

American College of Emergency Physicians (2011). Alternative methods to vascular access in the emergency department. Retrieved December, 2011 from http://www.acep.org/Content.aspx?id=80618\&terms=intraosseous\%20access.com.

American Heart Association, \& American Academy of Pediatrics (2011). Textbook of pediatric advanced life support. Dallas, TX: The American Heart Association.

Broselow, J. (2010). Broselow® Pediatric Emergency Tape. Armstrong Medical Company.

Cook Medical (2012). Cook manual intraosseous devices. Retrieved 2/17/2012 from http://www.cookmedical.com.

DeToledo, J. C., Minagar, A., Lowe, L., \& Merredith, R. M. (2002). Lidocaine-induced seizures in patients with history of epilepsy: Effects of antiepileptic drugs. Anesthesiology, 3, 737-739.

Drinker, C. K. (1922). The circulation in mammalian bone marrow. American Journal of Physiology, 62, 1-92.

Frascone, F. J., Jensen, J. P., Kaye, K., \& Salzman, J. G. (2007). Consecutive field trials using two different intraosseous devices. Prehospital Emergency Care, 11, 164-171.

Horton, M. A., \& Beamer, C. (2012). Powered intraosseous insertion provides safe and effective vascular access for pediatric emergency patients. Pediatric Emergency Care, 28(2), 185199.

Hoskins, S. L., Zacharia, B. S., Copper, N., \& Kramer, G. C. (2007). Comparison of intraosseous proximal humerus and sternal routes for drug delivery during CPR. Circulation, 116, II, 993. 
Infusion Nurses Society (2009). The role of the registered nurse in the insertion of intraosseous access devices. Journal of Infusion Nursing, 32(4), 187-188.

Jamshidi (product information). (2012).

Lamhaut, L., Dagron, C., Apriotesei, R., Gouvernaire, J., Elie, C., Marx, J. S., \& Carlie, P. (2010). Comparison of intravenous and intraosseous access by pre-hospital medical emergency personnel with and without CBRN protective equipment. Resuscitation, 81, 65-68.

Leidel, B. A., Kirchhoff, C., Braunstein, V., Biberthaler, P., \& Kanz, K. G. (2011). Comparison of intraosseous versus central venous vascular access in adults under resuscitation in the emergency department with inaccessible peripheral veins. Resuscitation, 8, [insert page numbers].

Merkel, S. I., Shayevitz, J. R., Voepel-Lewis, T., \& Malviya, S. (1997). The FLACC: A behavioral scale for scoring postoperative pain in young children. Pediatric Nursing, 23(3), 293-297.

Moran, L. R., Hossain, T., \& Insoft, R. M. (2004). Neonatal seizures following lidocaine administration for elective circumcision. Journal of perinatology, 24, 395-396.

Nutbeam, T., \& Daniels, R. (2010). ABCs of practical procedures. Oxford, U.K.: WileyBlackwell.

Philbeck, T. E., Miller, L. J., Montez, D., \& Puga, T. (2010). Hurts so good. JEMS, September, $59-68$.

Philbeck, T., Miller, L., \& Montez, D. (2009). Pain management during intraosseous infusion through the proximal humerus. Annals of Emergency Medicine, 54(3), S128. 
Vidacare (2012). EZ-IO intraosseous infusion system. Retrieved 4/15/2012 from http://www.vidacare.com.

Voigt, J., Waltzman, M., \& Lottenberg, L. (2008). Does intraosseous equal intravenous? A pharmacokinetic study. American Journal of Emergency Medicine, 26, 31-38.

Waismed (2012). Bone Injection Gun. Retrieved 4/15/2012 from http://waismed.com. 